\title{
Service-oriented mobile multimedia cooperative storing and delivery scheme based on opportunistic cloud coding and content-centric cloud compression
}

Nan Lin

\begin{abstract}
In order to improve the quality of multimedia communication and mobile users, the work efficiency of the cloud platform, the service-oriented mobile multimedia cooperative storing, and the delivery scheme were proposed based on opportunistic cloud coding and content-centric cloud compression. Firstly, according to the video frame and the multimedia content centric, the multimedia data compression algorithm was established based on time domain and frequency domain, which combined the image pixel scale and the component. Then, the opportunity cloud encoding program was proposed through the distortion scattering and the opportunity to cooperate with the cloud device control. Finally, the real-time transmission and cooperative feedback control of mobile multimedia streaming video content was proposed based on user requirements and service-driven demands. Experimental results show that the proposed scheme can provide high peak signal-to-noise ratio (PSNR) for large scale video frame transmission and keep high consistency and robustness from subjective experience to objective experience. In addition, the proposed scheme can significantly reduce the failure rate and improve the end-to-end communication performance.
\end{abstract}

Keywords: Mobile multimedia, Cooperative storing and delivery, Opportunistic cloud coding, Content-centric cloud compression

\section{Introduction}

In the mobile multimedia and cloud computing environment [1], the multimedia services [2] and user requirements are increasingly complex and rich. How to guarantee the quality of user experience and subjective evaluation of the quality of service has been the research hotspot for mobile multimedia applications. With the rapid development of mobile multimedia real-time services, the explosive growth of real-time video services based on cloud platform [3, 4] has emerged. The mobile multimedia streaming should provide reliable service through storage, forwarding, and distribution for the TV, PC, mobile phones, PAD, and other mobile users. In order to guarantee the quality of mobile multimedia services, it is urgent to solve the problems of storage, management,

Correspondence: linnanjs@163.com

College of Software Technology, Zhengzhou University, Zhengzhou, Henan 450002, China forwarding, and distribution of large capacity and high concurrent multimedia streaming services.

On the one hand, two scheduling algorithms were proposed in the article [5], which have been stored at a video server before transmission and are used to optimize the quality of scalable coded videos. The wear reduction techniques of phasechange random access memory (PRAM) were studied in the article [6] based on frame store (FS) in H.264 codec system. The store service operations practice course was developed in the article [7] based on simulation-based training of video clip instruction. The authors of article [8] generalized the existing class of immediately-decodable network coding schemes to take into account the deadline constraints.

On the other hand, about the multimedia delivery, the design and theoretical understanding of push-based content delivery was contributed in the article [9] for a converged broadcasting and cellular network, which was used to relieve the burden. An adaptive multimedia framework (ADAMS) was proposed in the article [10]

\section{Springer Open}

(c) 2016 The Author(s). Open Access This article is distributed under the terms of the Creative Commons Attribution 4.0 International License (http://creativecommons.org/licenses/by/4.0/), which permits unrestricted use, distribution, and reproduction in any medium, provided you give appropriate credit to the original author(s) and the source, provide a link to the Creative Commons license, and indicate if changes were made. 
for delivering scalable video and sensorial data to users. A holistic approach for supporting quality of experience (QoE)-aware multimedia content delivery to modern wireless networks was described by Aristomenopoulos et al. [11]. An optimal synthesis of a wideband log-periodic dipole array (LPDA) was introduced by Zaharis et al. [12], which could be performed under several requirements concerning the standing wave ratio and the forward gain over a wide frequency range.

In terms of encoding with cloud, a novel encoding procedure was proposed in the article [13] that resembles semi-random low-density parity-check (SR-LDPC) codes in both structure and performance. A proxy-based storage system for fault-tolerant multiple-cloud storage was presented in the article [14], which achieves costeffective repair for a permanent single-cloud failure. An effective inter-block multi-erasure coding scheme was designed in the article [15] for big bulk data transmission of cloud computing applications based on our prior research. Khalili et al. [16] considered the multistate radar set-up and distributed receive antennas, which were connected to a fusion center via limited-capacity backhaul links.

About research of coding and cloud computing, a new inter-level cloud compression architecture and algorithm was presented by Gupta et al. [17] which was suggested to implement inter-level compression algorithm. The effort to implement a recently proposed meshless dynamic cloud methadone modern high-performance graphic processing units with the compute unified device architecture (CUDA) programming model was established in the article [18]. Based on the uplink C-RAN systems considered by the authors of the article [19], a distributed frontal compression scheme was proposed at the distributed RRHs. The layered transmission and compression strategies were proposed in the article [20] that aim at opportunistically leveraging more advantageous channel conditions to neighboring base stations.

Under the existing research results, we study the following aspects: (1) the content-centric cloud compression scheme was designed based on the cloud platform and multimedia stream content; (2) the opportunistic cloud coding scheme was established according to the cloud scale and the pixel scale of three components in the frequency domain; and (3) the service-oriented mobile multimedia storing and cooperative delivery scheme was proposed for guaranteeing the real-time transmission and cooperative feedback control of the mobile multimedia streaming.

The rest of the paper is organized as follows. Section 2 describes the content-centric cloud compression scheme. In Section 3, we design the opportunistic cloud coding scheme. In Section 4, we proposed the service-oriented mobile multimedia storing and cooperative delivery scheme. Experiment results are given in Section 5. Finally, we conclude the paper in Section 6.

\section{Content-centric cloud compression scheme}

Assume that the multimedia data includes $N$ video frames. Video frame pixel of each video frame is expressed as $\operatorname{px}(i, j)$. Parameter $i$ belongs to interval $[0, X]$. Parameter $j$ belongs to interval [0,Y]. Here, $X$ is the pixel number of horizontal direction video frame. $Y$ is the pixel number of vertical direction video frame. $\operatorname{TF}(i, j)$ denotes the signal content of multimedia data. Multimedia data content $\mathrm{MC}$ based on time domain and frequency domain is shown by formula (1).

$$
\mathrm{MC}=N \frac{\sum_{i=0}^{X} \sum_{j=0}^{Y}|\mathrm{TF}(i+1, j+1)-\mathrm{TF}(i, j)|^{2}}{\prod_{i=0}^{X} \mathrm{px}(i, Y)^{N * X}}
$$

Based on multimedia data compression of the cloud platform, we considered the time domain and frequency domain conversion. The compression content is given by formula (2).

$$
\left\{\begin{array}{l}
\mathrm{MCC}=\int_{i=0}^{N} \mathrm{px}\left(t_{x}, f_{y}\right) \mathrm{dt} \\
t_{x}=\sqrt{\frac{1}{X} \sum_{i=0}^{X} \sum_{j=0}^{Y}\left|t_{i}-t_{j}\right| \frac{X+Y}{N}} \\
f_{y}=\frac{Y}{N} \prod_{t=0}^{X} \sqrt{\left|f_{t}-f_{t+1}\right|}
\end{array}\right.
$$

Here, $t_{x}$ is the delay jitter. $t_{y}$ is the frequency domain conversion. It is well known that the frequency domain and time domain have a great influence on the efficiency and quality of multimedia compression in the cloud platform.

Encoding rate is $K$ pixels per second. The multimedia data for cloud encoding can obtain space gain, as shown in formula (3).

$$
\left\{\begin{array}{l}
S_{G}=\left|T F-\sum_{i=0, j=0}^{X, Y} \operatorname{px}(i, j)\right| \frac{S_{\mathrm{MC}}}{S_{\mathrm{MCC}}} \\
S_{\mathrm{MC}}=\sum_{i=0}^{X} \sum_{j=0}^{Y}|\mathrm{TF}(i+1, j+1)-\mathrm{TF}(i, j)|^{2} \\
S_{\mathrm{MCC}}=\sum_{j=0}^{Y} \sum_{i=j+1}^{X} \int_{i=0}^{N} \operatorname{px}\left(t_{x}, f_{y}\right)
\end{array}\right.
$$

Here, $S_{G}$ is the space gain. $S_{\mathrm{MC}}$ is the cloud platform space occupancy before the compression of multimedia 
data. $S_{\mathrm{MCC}}$ is the space occupancy of cloud platform after compression of multimedia data.

Thus, the distortion $D_{t}$ is caused by the frequency domain conversion and the distortion $D_{f}$ caused by the frequency domain conversion. The delay jitter $D_{\text {IS }}$ is shown in the formula (4), which was caused by the multimedia data compression.

$$
D_{\mathrm{IS}}=\frac{D_{t}}{D_{f}}
$$

The influence of multimedia data content on the correlation between the time and frequency domain was reflected by the distortion ratio. In the process of cloud compression, the content of multimedia data is optimized by analyzing the changes of $D_{\mathrm{IS}}, D_{t}$, and $D_{f}$. Therefore, the distortion of each video frame in the multimedia data can be calculated by the formula (5).

$$
\left\{\begin{array}{l}
D_{F}(x, y)=\frac{\mathrm{TF}\left(\mathrm{VF}_{x, y}\right)}{C_{c l} \delta^{\sqrt{N}} \prod_{i=0}^{X+Y} \mathrm{px}_{i}} \\
\mathrm{VF}_{x, y}=\prod_{i=x}^{X} \sum_{j=y}^{Y} \mathrm{px}(i, j)
\end{array}\right.
$$

where, $D_{F}(x, y)$ denotes the multimedia video data frame distortion value. $\mathrm{VF}_{x, y}$ expresses multimedia content of these video frames. $\delta$ is the variance of $D_{\mathrm{IS}}$ on the time and frequency domain. $C_{\mathrm{cl}}$ denotes the cloud compression ratio.

Combining Eqs. (1) to (5), the multimedia compressed content $C_{\mathrm{MC}}$ is given by Eq. (6), which depended on the conversion jitter of cloud platform multimedia data in time domain and frequency domain.

$$
\left\{\begin{array}{l}
C_{\mathrm{MC}}=\frac{\sum_{j=0}^{Y} \mathrm{TF}(X, j) \int_{0}^{+\infty} \mathrm{px}(N, y) \mathrm{dy}}{\delta^{2} \sum_{i=0}^{X} \mathrm{DF}(x, Y) \sum_{j=0}^{Y} \mathrm{TF}(X, y)} \\
\operatorname{px}(N, Y) \geq \prod_{i=x}^{X} \sum_{j=y}^{Y} \mathrm{px}(i, j)
\end{array}\right.
$$

In the cloud compression, a frequency domain of transition distortion gain could be obtained based on three different frequency directions. How to select three frequency directions was shown in Fig. 1. The progress of quantifying and reconstructing residual image samples of content-centric multimedia data compression is equivalent to the object pixel iteration of the video frames. The video frame reconstruction based on pixel expansion can judge the correlation of the subsequent pixel, which is used to provide reference pixels for cloud compression. It is well known that the multimedia data correlation of adjacent clouds is strong. Therefore, the combination of the multimedia stream video frames and frame reconstruction pixels would have better multimedia compression performance.

When we compressed the content-centric multimedia data, the expanding of the pixel would be as the reference compression pixel. The filtering progress can make up the video frame pixel distortion caused by compression. The edges fuzzy extension model of the video frame could reduce the cloud compression

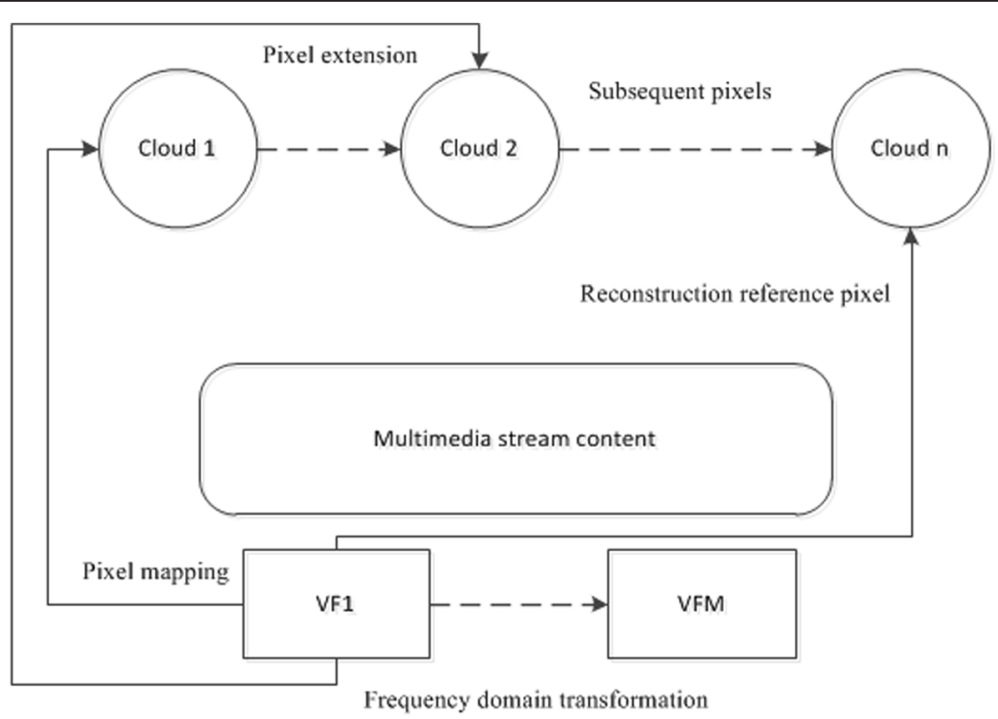

Fig. 1 Content-centric cloud compression architecture of frequency domain transformation 
space complexity. In cloud compressed video frames, the cooperative filtering of cloud equipment can denoise the multimedia stream, which could obtain more cloudy compression gain. On the three different frequency directions in Fig. 1, according to content requirements, we selected the best cloud platform resources as a follow-pixel reference pixel in order to increase the cloud compression weight. Workflow is shown in Fig. 2.

\section{Opportunistic cloud coding scheme}

The multimedia data coding of cloud devices will have a certain quality of residuals. These residuals will be distributed to multiple cloud passed, so that the distortion scattered into the encoder. Distortion scattering process can be represented as a cloud platform to solve the quality residual and quantify residual process. Assume that cloud-platform multimedia streaming video frame matrix $\mathrm{VF}=\left(\mathrm{VF}_{i j}\right) X^{*} Y$. Transmission quality resulting residual matrix $\mathrm{RQ}=\left(\mathrm{RQ}_{i j}\right) X^{*} Y$. Distortion scattering matrix $\mathrm{DS}=\left(\mathrm{DS}_{i j}\right) X^{*} Y$. Quantization matrix coding residual cloud platform $\mathrm{CEQS}=\left(F_{i j}\right) X^{*} Y$. The above matrixes have the following relations shown as formula (7).

$$
\left\{\begin{array}{l}
\mathrm{VF}=\frac{N}{C_{N}} \sum_{i=0, j=0}^{X, Y}\left(\frac{\mathrm{VF}_{i j}}{\mathrm{RQ}_{i j}}\right)_{X * Y} \\
\mathrm{CEQS}=\mathrm{VF} \sum_{i=0}^{X} \sum_{j=0}^{Y} F_{i j}\left(\frac{\mathrm{DS}_{i j}}{C_{N}}\right)
\end{array}\right.
$$

Here, $C_{N}$ is the umber of the active cloud in cloud platform. From formula (7), there is a direct relationship between data quality reference residuals, cloud size, video frame size, and image pixel size of the cloud encoded multimedia.
After the matrix form of multimedia streaming video frame, matrix VF is expanded as Eq. (8).

$$
\left\{\begin{array}{l}
\mathrm{VF}=\left[\begin{array}{ccc}
\mathrm{VF}_{00} & \cdots & \mathrm{VF}_{0 j} \\
\vdots & \ddots & \vdots \\
\mathrm{VF}_{i 0} & \cdots & \mathrm{VF}_{i j}
\end{array}\right]_{X * Y}\left[\begin{array}{ccc}
\mathrm{px}_{00} & \cdots & \mathrm{px}_{0 j} \\
\vdots & \ddots & \vdots \\
\mathrm{px}_{i 0} & \cdots & \mathrm{px}_{i j}
\end{array}\right]\left[\begin{array}{l}
a_{0} \\
\vdots \\
a_{j}
\end{array}\right] \\
a_{i}=\cos \left(\frac{\pi}{2}-\theta\right) \sqrt{\frac{\mathrm{VF}_{i Y}}{C_{N}}}
\end{array}\right.
$$

Here, $a_{i}$ denotes cloud coding opportunistic weights of the pixel array. Cloud sum of the number of pixels in the horizontal direction is divided by the forwarded video frames, taking into account the vertical angle $\theta$ of the transmitting antenna cloud device.

When forwarding interactive multimedia streams between multiple clouds, clouds would opportunistically receive or forward the multimedia stream. Multimedia data packets of the video frame and the pixel array are from different clouds. There are the quality visual correlation consistencies between the cloud platform encoding and multimedia playback source packet stream. Related VRM visual mapping process was shown in Eq. (9).

$$
\left\{\begin{array}{l}
\mathrm{VRM}=\int_{i=0}^{\infty} \exp \left(\frac{N}{C_{N}^{X}}\right) \mathrm{px}^{Y} \mathrm{dpx} \\
\mathrm{VF}_{X}^{1}=|\mathrm{TF}(1, X-1)-\mathrm{TF}(1, X-2)| \sqrt{\left|D_{t}^{2}-D_{f}^{2}\right|} \\
\mathrm{VF}_{X}^{2}=|\mathrm{TF}(Y-1,1)-\mathrm{TF}(Y-1, X-2)| \sqrt{\left|D_{t}^{2}-D_{f}^{2}\right|} \\
\mathrm{VF}_{X}^{3}=\left|\mathrm{VF}_{X}^{2}-\mathrm{VF}_{X}^{1}\right| \sqrt{\left|D_{t}^{2}-D_{f}^{2}\right|} \mid \sum_{j=0}^{Y} \mathrm{TF}_{j}
\end{array}\right.
$$

Here, vision-related objects are mapped in pixels scattering iterative. $\mathrm{VF}_{X}^{1}$ denotes the collection of video frames extending direction of the pixel frequency

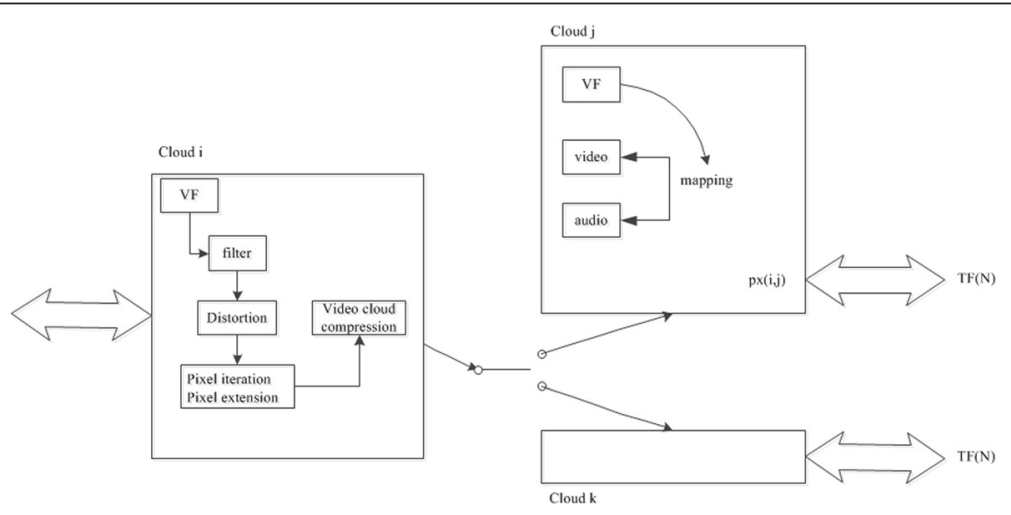

Fig. 2 Workflow of the content-centric cloud compression scheme 
domain. $\mathrm{VF}_{X}^{2}$ denotes the pixel development orientation video frame set the frequency domain. $\mathrm{VF}_{X}^{3}$ denotes the subsequent video frame pixels belong collection biorthogonal $\mathrm{VF}_{X}^{1}$ and $\mathrm{VF}_{X}^{2}$.

In summary, the opportunity to process multimedia stream encoded cloud data was shown in Fig. 3.

With the content-centric cloud compression, the transition switching delay jitter and frequency domain frequency domain coding can be obtained at the opportunistic cloud coding, which could eliminate the cloud and weaken the working principle shown in Fig. 4.

In order to strengthen opportunities for cloud encoding algorithm robustness of $R$ (OCC) and optimized multimedia streaming compression cloud platform complexity SC (CC), using the formula (10), the method of cloud distributed multimedia video frame quantization and coding gradient, thus the best multimedia stream video frame matrix and pixel mapping matrix.

$$
\left\{\begin{array}{l}
R(\mathrm{OCC})=\mathrm{RC}_{S} \mathrm{VF}_{D} \sum_{i} \frac{\sum_{j} \mathrm{PK}_{j}}{\mathrm{VF}_{i}} \\
\mathrm{SC}(\mathrm{CC})=\sum_{i} \mathrm{VF}_{i}\left\|\mathrm{px}_{i}\right\|^{2}
\end{array}\right.
$$

Here, $\mathrm{RC}_{S}$ is the correctly received probability of encoded video frame. $\mathrm{VF}_{D}$ is the transmission delay of video frames. The robustness could be analyzed based on the encode pixels and video frames.

\section{Service-oriented mobile multimedia storing and cooperative delivery scheme}

We researched the multimedia storing and cooperative delivery scheme of mobile multimedia for multimedia applications cloud platform. According to the integration background of cloud platform and mobile Internet, the mobile multimedia streaming video content file would be transferred and cooperatively controlled based on the user requirements and service-driven demands. Resources of multimedia applications are managed based on the service-driven cloud platform. The resource management scheme combined the multimedia content and service-driven scheme of cloud platform, which could globally optimize cloud server storage space and opportunistically forward multimedia stream. Resource information would be distributed to the cloud users, who could obtain the cloud service guarantee and play media with pixel mapping servers. Based on the service-driven scheme and multimedia streaming content, the required address list of the long time and large-scale multimedia streaming could be obtained from the multimedia opportunistic communications cloud platform. And thus, the users would feedback the opportunistic code request and content-centric multimedia compression request to the cloud platform. In order to protect the service from the multimedia streaming content management, cloud platform should optimize the multimedia streaming server through the entire network. The above process is shown in Fig. 5.

Figure 5 gives a mobile Internet-based cloud platform multimedia streaming content distribution and storage architectures. A different root node ID corresponds with the multimedia stream, which would be assigned to the multimedia streaming content and distributed multimedia streaming service data. The wide-area storage content-centric multimedia data fragmentation and video pixel block would be compressed. Compressed data and multimedia streaming ID are also stored in the root of the boot partition. Meanwhile, the users request information should be preserved. Computer terminals of

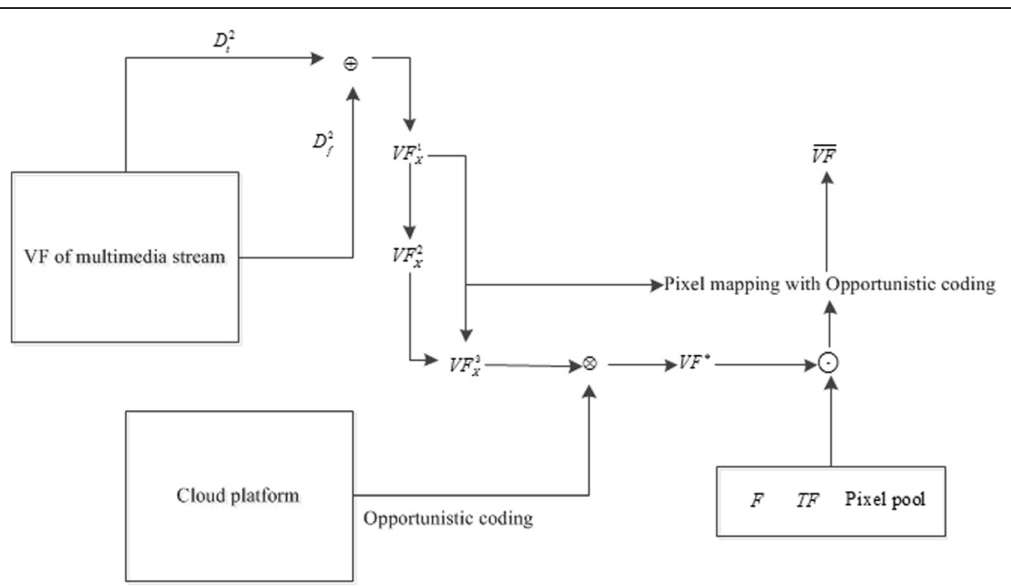

Fig. 3 Video frame workflow of opportunistic cloud coding scheme 


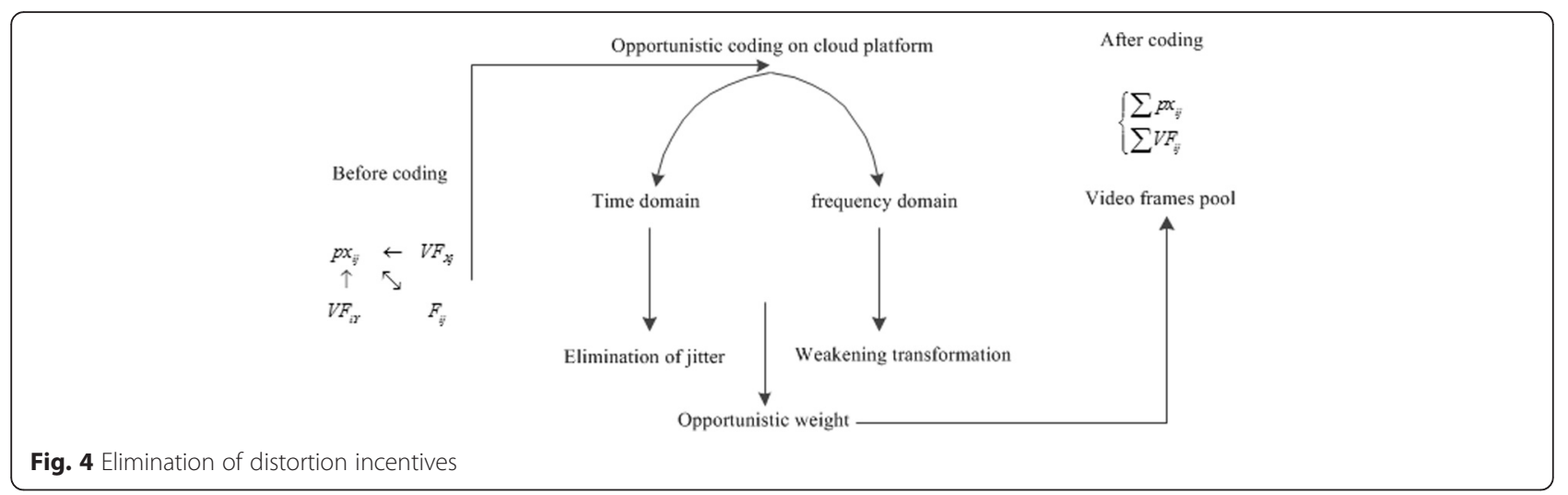

cloud platforms are deployed according to the $x$ line and $y$ column. When multimedia data reached the cloud platform, the cooperative computing progress would be executed from $(1,1)$ terminal to $(\mathrm{X}, \mathrm{Y})$ terminal.

Cloud would find the optimal opportunistic cloud coding video frame through the root of WAN cloud storage platform. The opportunistic subsequent cloud pixel size and continuous mapping video frame size can be obtained by formulas (8) and (9), respectively. The mapping relationship between the root node and distribution node would be impacted by the pixel size and video frame size. Multimedia streaming block file and cloud platform distribution nodes would be decided by the pixel mapping matrix or the root node mapping table.

In order to ensure the rationality of the cloudplatform multimedia streaming content and forward balance of content delivery, we should pay attention to the following points. Meanwhile, the following points could ensure the service-driven balance and high cloud platform resource utilization.
(1)Wide storage management server distributes the reference matrix, which could help to make cloud platform multimedia stream pixel partition and video frames partition scheme. The scheme could select the best multimedia streaming data unit.

(2)Cloud platform establishes the corresponding multimedia streaming as the cloud cooperative distribution reference matrix based on the service drive. The multimedia distribution data unit and a cloud platform ID would be forwarded to WAN root node.

(3) The appropriate layout file of distribution cloud platform would be obtained through the distribution data unit and clouds ID. Meanwhile, the required cloud scale would be calculated cooperatively in the cloud platform. And then a two-dimensional layout of the cloud device would be designed. If the root storage matrix is not consistent with the multimedia platform distributed information, we have to consider the following five points.

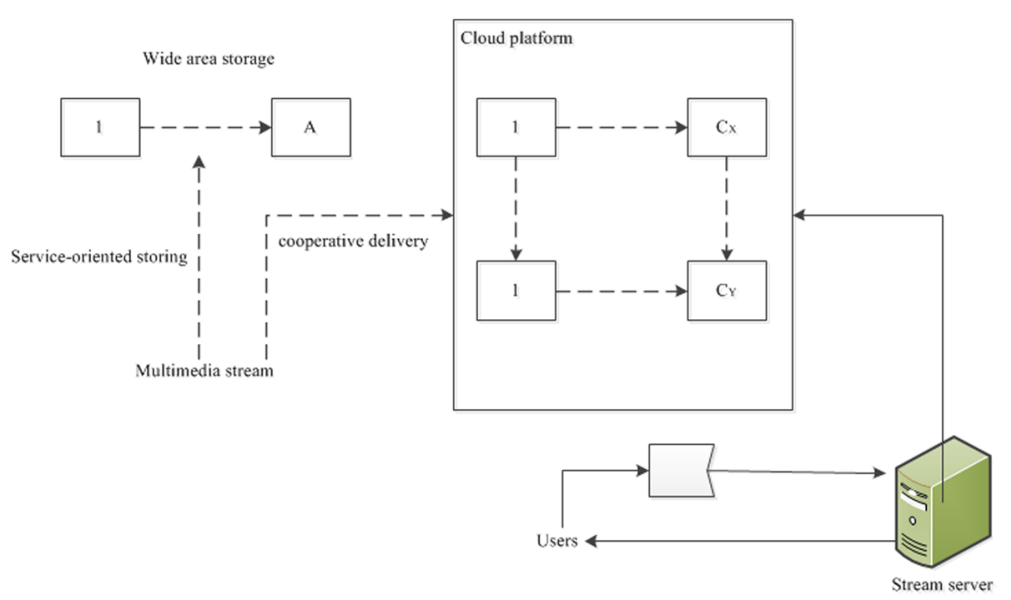

Fig. 5 Architecture of service-oriented mobile multimedia cooperative storing and delivery 
(4) Cloud device is activated to optimize the root layout, combined with a multimedia stream information distribution. Could service would access to multimedia streaming content from a wide area network storage server. Cloud code must follow the service drive mapping requirements.

(5) Parameters of clouds multimedia compression and encoding scheme would be updated through cooperative computation. The next round multimedia streaming video frames and partitions pixels clusters would be selected through expanding pixels and the specified frequency block.

(6)Cloud platform should inform the wide-area storage server root node and streaming media server.

(7)Cloud platform activates the cloud matrixes and multimedia streaming media servers for forwarding the cloud data. User service request would be integrated into multimedia cloud platform distribution scheme through the cooperative computation.

(8)Client feedback the service driver updating request according to the quality of multimedia broadcast and subjective evaluation. Then, the next round of multimedia cloud transport would be active.
The opportunistic cooperative management system consists of $K$ storage servers, $N$ video frames, $\mathrm{CN}$ a cloud and $M$ mobile node, and a wide-area network storage server cluster components. Figure 6 is a layered architecture of distributed cooperative opportunities management system. Six cloud-like paintings on behalf of the cloud platform multimedia distribution source site. Dot represents WAN storage server nodes in the cluster. Diamonds represent multimedia streaming video frame group. Some WAN cloud storage service node and cloud nodes form a distributed platform opportunities for synergies hierarchical management system.

In this hierarchical management system, all of the mobile node information and multimedia content information would be stored in the root node of WAN storage server. Multimedia mapping information includes multimedia streaming content size, video frame size, pixel matrix, storage space utilization and distortion ratio, and other information. The above information is the basis of the content-centric cloud compression and reference weights chance cloud encoding. Cloud platform also includes multimedia streaming compression and streaming server mapping content between decode multimedia content.

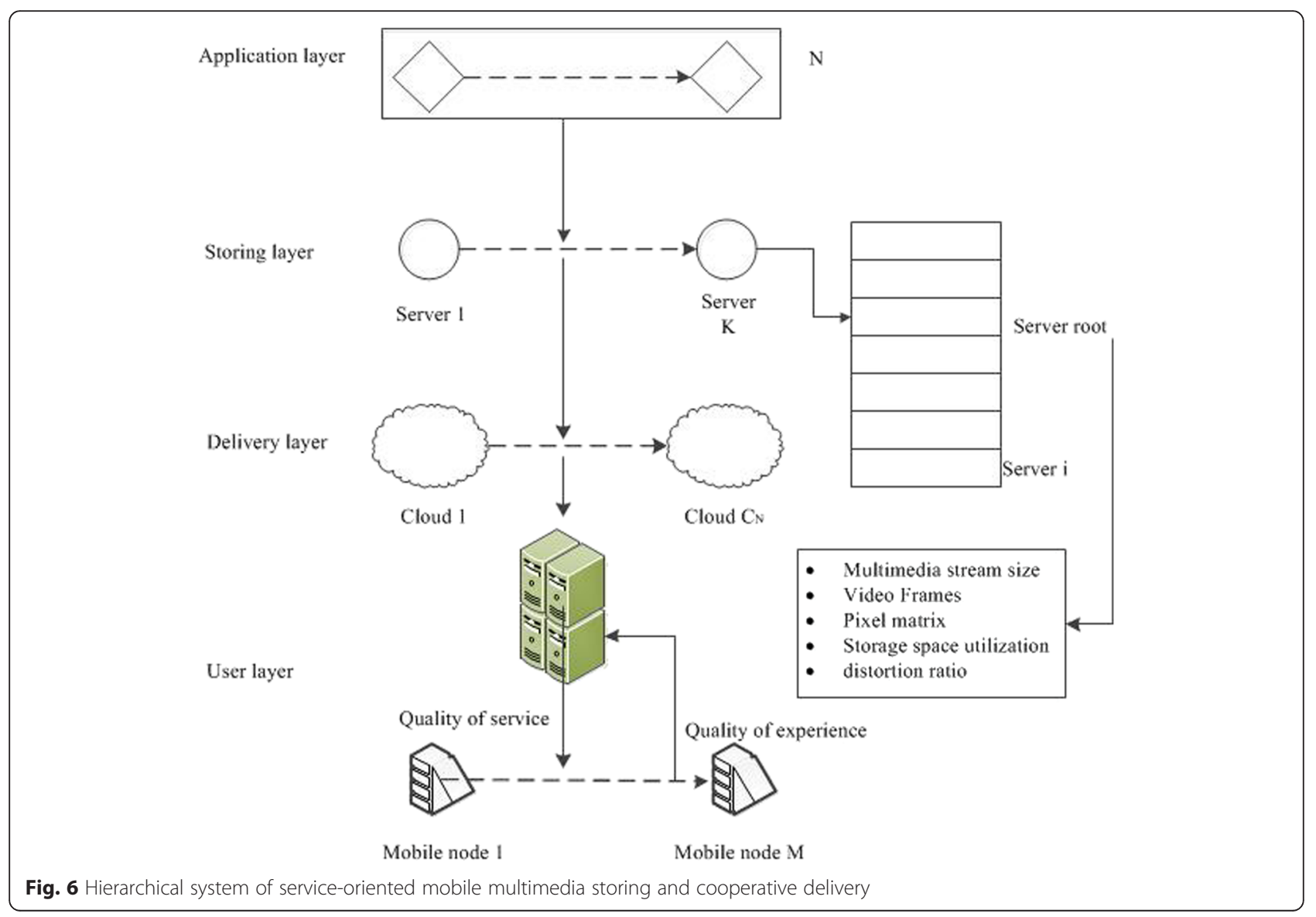




\section{Performance verification and comparison}

To verify the visual performance and service quality assurance of the proposed scheme denoted as SMCTD-OCC, we set up three experiments. We chose some traffic congestion periods pedestrians, bicycles and cars in urban traffic roads intersect actual background traffic video clip slowly forward, including the 1000 video frames. Traffic camera captured video in real-time transfer after WAN storage server. Users could access live video through mobile multimedia access cloud platform. We compared the difference between compression and video quality and the actual quality of the encoded video from the subjective and objective quality.

The working process of experiment 1 is as follows.

(1)We select the complex part of the video frame.

These frames are distributed through a wide-area network storage server. Operation results and multimedia content would be mixed together. The integration of the results would be sent to the cloud platform.

(2)The simple traffic video frames would be embedded in the stack location of subsequent cloud compressed video frames. Then, the decompressed reference video frame would be obtained.

(3) The PSNR of cloud compression and cloud encoding video in three directions could be calculated by Eqs. (1) to (6) and (7) to (10), respectively. We compared the average PSNR value and the source video frame PSNR. The analysis of video quality objective difference after cloud platform transmission is shown in Fig. 7.

(4) The multimedia video quality subjective evaluation was given based on the actual viewing quality of mobile users. The 20 who experienced move

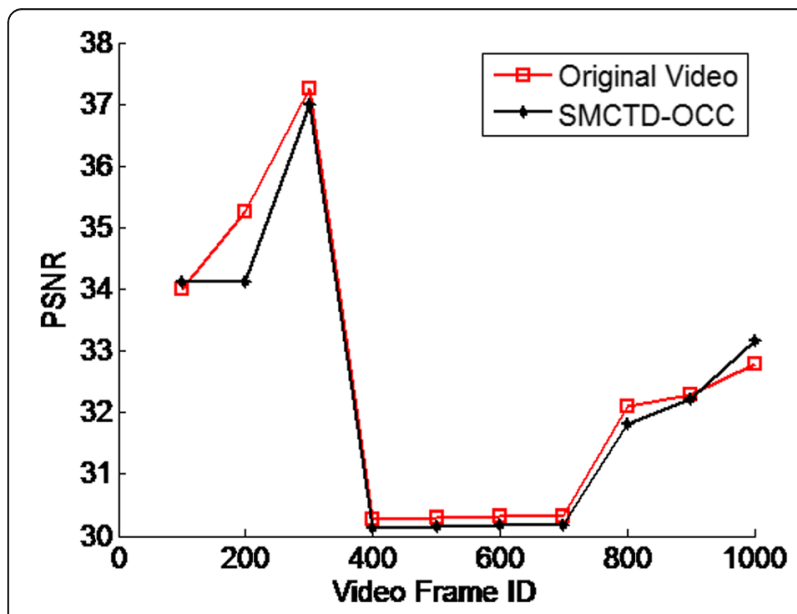

Fig. 7 PSNR comparison randomly and used mobile phones, laptops, and in-car video systems simultaneously. They watched a video at the same lighting conditions, the score results was shown Table 1.

From Fig. 7 and Table 1, the performance of proposed scheme SMCTD-OCC is close to the actual video content. These are because that the proposed cloud compression scheme is distributed cloud computing based on the content of the source video. Pixel extending, expanding, and subsequent reference come from different cloud devices. While maintaining the high cloud computing devices more efficient, it reduces the complexity of cloud video transmission equipment and distortion probability. Whether simple or complex background video background video at the same computing resources, through three direction frequency domain components interact with the fusion of compressed video compressed video source video from the subjective and objective are to maintain a high consistency and robustness.

Experiment 2 analyzed the compressed and encoded multimedia streaming content deterministic structure, compressed video frame structure, coding matrix, and the user mobility affects the video frame transmission in-depth. Valid video frame could be obtained through three directional components in the frequency domain of a multimedia data storage and distribution properties. Specific process is as follows:

(1)We choose the video frame similarity of complex background content, and a simple background is higher than $85 \%$. Linear relationship between compression efficiency and distortion would be counted by background subtraction approach.

(2) Statistical distortion video frame contains a video clip, and the client cannot normally play video clips, and there is significant background partial illumination changing video frames that are compared, obtained after the XOR video frame and the video frame reference for statistical failure value.

Figure 8 shows the failure of the frame rate statistics. Whether at a high speed or low-speed node

Table 1 Subjective evaluation

\begin{tabular}{llllll}
\hline User type & $V^{1}$ & $V^{2}$ & $V^{3}$ & $\begin{array}{l}\text { General } \\
\text { comments }\end{array}$ & $\begin{array}{l}\text { Ratio of high } \\
\text { quality users (\%) }\end{array}$ \\
\hline Mobile phone & 37 & 35 & 34 & Consistency & 83 \\
Laptop & 36.5 & 33.8 & 34.2 & Consistency & 85 \\
Vehicle video system & 35.8 & 34 & 33.5 & Consistency & 87 \\
\hline
\end{tabular}




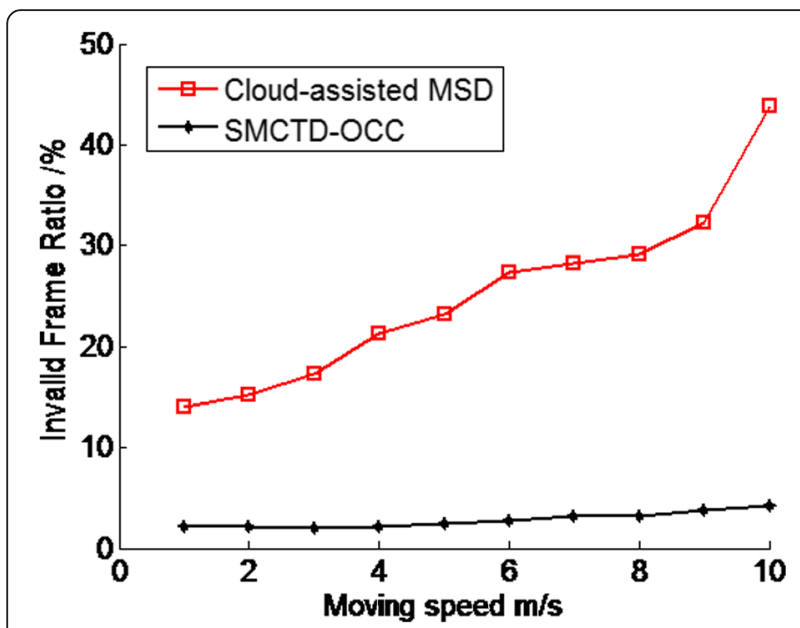

Fig. 8 Invalid frame ratio

moving scenes, the video playback quality of the proposed program SMCTD-OCC has been increased significantly, which is better than one of cloud-assisted MSD. With the increase of node mobility rate, frame rate change proposed scheme failed SMCTD-OCC stabilize. The cloud-assisted MSD showing speed upward trend.

In experiment 3 , the end-to-end delay and decodable frame ratio of the proposed scheme SMCTDOCC statistics-based and client-end transmission delay cloud platform storage and distribution mechanisms cloud-assisted MSD were obtained from sending video of traffic cameras to the user. The results are shown in Fig. 9. About the cloud-assisted MSD, the multimedia data storage and distribution platform focused on the cloud, which leaded to the increase of cloud equipment workload and decrease of the multimedia data mining accuracy and efficiency. Thereby, the transmission delay increased obviously, and the packet loss rate is more serious. Hence, the client playback quality is very poor.

\section{Conclusions}

Based on the study of multimedia streaming content classification, cloud platform architecture and user mobility characteristics, we proposed the serviceoriented mobile multimedia cooperative storing and delivery scheme with the storage of multimedia data, distribution, and encoding after layered processing. Multimedia data is stored in a wide-area network storage server. And each multimedia stream records information to the root node. Multimedia compression would be completed in the cloud platform. We regarded the video frame as the research object and the multimedia content as the center. Then combining the image pixel scale and the component, a multimedia data cloud compression algorithm was established based on time domain and frequency domain. When the compression of multimedia distribution activated state of the cloud devices in the cloud platform, the multiple cloud devices streaming transmission progress would produce a certain amount of quality residuals, through false scattering and cloud devices opportunistic control. Then the opportunistic cloud encoding program was proposed. In order to reduce the distortion rate and optimize the transmission quality of the video frame, we proposed realtime transmission and cooperative feedback control scheme of the mobile multimedia streaming video content file based on user needs and service driven, which also could improve the user experience quality and cloud platform work efficiency. Three groups of experiments respectively demonstrate the validity and feasibility of the proposed scheme from the subjective and objective multimedia quality evaluation, video frame distortion, end-to-end transmission delay and decoding frame rate. a

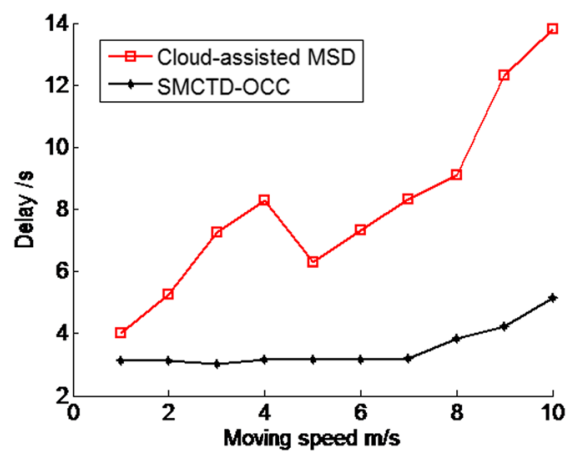

b

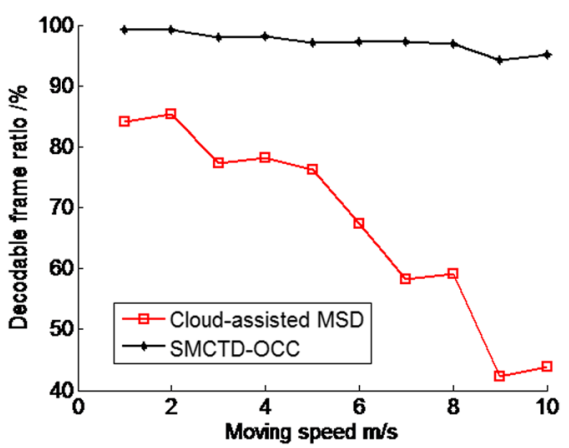

Fig. 9 a Delay. b Decodable frame ratio. Performance analysis of end-to-end 


\section{Competing interests}

The author declares that he has no competing interests.

\section{Acknowledgements}

This work is supported in part by the National SME Technology Innovation Fund Project (ID: 10C26214102198).

Received: 14 August 2015 Accepted: 9 June 2016

Published online: 23 June 2016

\section{References}

1. G Cheung, A Ortega, N-M Cheung, Interactive streaming of stored multiview video using redundant frame structures. IEEE Trans. Image Process. 20(3), 744-761 (2011)

2. Y Yun, C Song, N Lin et al., Cross-layer design for delay-and energyconstrained multimedia delivery in mobile terminals. IEEE Wirel. Commun. 21(4), 62-69 (2014)

3. A Cosimo, G Rossano, G Marco, Exploiting rateless codes in cloud storage systems. IEEE Trans Parallel Distributed Syst 26(5), 1313-1322 (2015)

4. C Yang, X Zhang, C Zhong et al., A spatiotemporal compression based approach for efficient big data processing on cloud. J. Comput. Syst. Sci. 80(8), 1563-1583 (2014)

5. C Chen, RW Heath, AC Bovik et al., A markov decision model for adaptive scheduling of stored scalable videos. IEEE Trans Circuits Syst Video Technol 23(6), 1081-1095 (2013)

6. G Sanchuan, L Zhenyu, L Guohong et al., Content-aware write reduction mechanism of $3 \mathrm{~d}$ stacked phase-change RAM based frame store in H.264 video codec system. IEICE Trans. Fundam. Electron. Commun. Comput. Sci. E96-A(6), 1273-1282 (2013)

7. C-H Lin, Y-R Yen, W Pai-Lu, Utilizing simulation-based training of video clip instruction for the store service operations practice course. Int J Distance Educ Technol 13(4), 98-107 (2015)

8. X Li, C-C Wang, X Lin, On the capacity of immediately-decodable coding schemes for wireless stored-video broadcast with hard deadline constraints. IEEE J Selected Areas Commun 29(5), 1094-1105 (2011)

9. K Wang, Z Chen, H Liu, Push-based wireless converged networks for massive multimedia content delivery. IEEE Trans. Wirel. Commun. 13(5), 2894-2905 (2014)

10. Z Yuan, G Ghinea, G-M Muntean, Beyond multimedia adaptation: quality of experience-aware multi-sensorial media delivery. IEEE Trans Multimedia 17(1), 104-117 (2015)

11. A Georgios, K Vassilios, K Giannis, et al. Experimentally driven quality of experience-aware multimedia content delivery in modern wireless networks. Int. J. Commun. Syst. 2015.

12. ZD Zaharis, C Skeberis, PI Lazaridis, et al. Optimal wideband LPDA design for efficient multimedia content delivery over emerging mobile computing systems. IEEE Syst. J. 2015.

13. R Sun, X Cai, J Liu et al., Distributed SR-LDPC codes over multiple-access relay channel and its applications in cloud storage. Concurrency Comput 27(8), 2064-2077 (2015)

14. H Chen, Y Hu, PPC Lee et al., NCCloud: a network-coding-based storage system in a cloud-of-clouds. IEEE Trans. Comput. 63(1), 31-44 (2014)

15. S Pei, G Chen, S Zhang et al., Inter-block multi-erasure coding scheme for cloud-based big bulk data transmission. J. Internet Technol 15(6), 1013-1023 (2014)

16. S Khalili, O Simeone, AM Haimovich, Cloud radio-multistatic radar: joint optimization of code vector and backhaul quantization. IEEE Signal Process Letters 22(4), 494-498 (2015)

17. PKD Gupta, S Pattnaik, M Nayak, Inter-level spatial cloud compression algorithm. Def. Sci. J. 64(6), 536-541 (2014)

18. $\mathrm{ZH} \mathrm{Ma}, \mathrm{H}$ Wang, $\mathrm{SH} \mathrm{Pu}, \mathrm{A}$ parallel meshless dynamic cloud method on graphic processing units for unsteady compressible flows past moving boundaries. Comput. Methods Appl. Mech. Eng. 285, 146-165 (2015)

19. X Rao, VKN Lau, Distributed fronthaul compression and joint signal recovery in cloud-RAN. IEEE Trans. Signal Process. 63(4), 1056-1065 (2015)

20. S-H Park, O Simeone, O Sahin et al., Robust layered transmission and compression for distributed uplink reception in cloud radio access networks. IEEE Trans. Veh. Technol. 63(1), 204-216 (2014)

\section{Submit your manuscript to a SpringerOpen ${ }^{\circ}$ journal and benefit from:}

- Convenient online submission

- Rigorous peer review

- Immediate publication on acceptance

- Open access: articles freely available online

- High visibility within the field

- Retaining the copyright to your article 